\title{
Pair production with electron capture in peripheral collisions of relativistic heavy ions
}

\author{
C.A. Bertulani ${ }^{(a, b, *)}$ and D. Dolci ${ }^{(a)}$ \\ ${ }^{a}$ Instituto de Física, Universidade Federal do Rio de Janeiro, \\ 21945-970 Rio de Janeiro, RJ, Brazil \\ ${ }^{b}$ Brookhaven National Laboratory, Upton, NY, USA
}

(November 11, 2018)

\begin{abstract}
The production of electron-positron pairs with the capture of the electron in an atomic orbital is investigated for the conditions of the Relativistic Heavy Ion Collider (RHIC) and the Large Hadron Collider LHC). Dirac wave functions for the leptons are used, taking corrections to orders of $Z \alpha$ into account. The dependence on the transverse momentum transfer is studied and the accuracy of the equivalent photon approximation is discussed as a function of the nuclear charge.
\end{abstract}

PACS numbers: 25.75.Dw,25.30.Rw

\section{INTRODUCTION}

Peripheral collisions with relativistic heavy ions are a source of several interesting physical processes and have attracted a great number of theoretical and experimental work (see, e.g., [1], and references therein). One of the processes of interest is pair production by the strong electromagnetic field of large $\mathrm{Z}$ nuclei in relativistic heavy ion colliders. In the case of production of free pairs the theoretical interest resides in the non-perturbative character of the process. Different techniques have been used to study this problem theoretically, but we will not discuss them here. Another case of general interest is the production of pairs in which the electron is captured in a bound state of one of the colliding nuclei [2] (see also ref. [3] and references therein). The interest here arises from its consequence for luminosity changing of the relativistic ion beams in colliders like RHIC (Relativistic Heavy Ion Collider) at Brookhaven, and the LHC (Large Hadron Collider) at CERN (for a recent analysis, see [4]). A more striking application of this process was the recent production of antihydrogen atoms using relativistic antiproton beams [5]. In that case the positron is produced and captured in an orbit of an antiproton.

Early calculations of pair production with capture used perturbation theory [6,2], the only difference being the way the distortion effects for the positron wavefunction were taken into account. Evidently the calculation is best performed using the frame of reference of the nucleus where the electron is captured. Many other calculations have been performed $[7$ 15. Some of them used non-perturbative approaches, e.g., coupled-channels calculations. However, in contrast to the production of free pairs, pair-production with atomic capture of the electron is well described in perturbation theory. The large coupling constant for large nuclei $(Z \alpha \sim 1)$ does not matter here, since the scaled matrix element divided by $Z \alpha$, i.e. $M /(Z \alpha)$, is much smaller in this case than it is for the production of free pairs. In other words, the overlap between the positron and the electron wavefunctions is much less than that for the production of free pairs. The first terms of the perturbation series are small enough to neglect the inclusion of higher order terms [2]. Indeed it was explicitly shown in ref. [15] that non-perturbative calculations modify the cross sections at most by $1 \%$.

The theoretical difficulty here is to properly account for the distortion of the positron wavefunction in the field of the nucleus where the electron is captured. In this article we study this property more closely, and compare our results with the calculations of ref. [2], which were based on the Sommerfeld-Maue wavefunctions for the positron [18]. We also investigate the accuracy and limitations of the equivalent photon approximation (EPA), what will gives us a clear understanding of the effects of distortion. We have closely used the technique of ref. [17] for the study of 
anti-hydrogen production cross sections at LEAR/CERN. We show that a commonly used approximation obtained in ref. [2] yields results up to a factor 5 too small for large ion charges.

In section 2 we deduce the cross sections for pair production with K-shell capture and obtain the cross sections in terms of longitudinal and transverse components. In section 3 we compare the calculation with that of pair production with capture by real photons. This allows us to disclose the role of distortion effects. We also show that the transverse part of the virtual photon cross section is much larger than the longitudinal one. In section 4 we calculate total cross sections and compare them to the results obtained in ref. [2], to the equivalent photon approximation and to recent experimental data. Our conclusions are given in section 5 .

\section{PAIR PRODUCTION WITH K-SHELL CAPTURE}

In the semiclassical approach we assume that the relativistic ions move along straight lines. Although the laboratory system is a more symmetric frame of reference, the calculation is best accomplished in the frame of reference of one of the ions - the one in which the electron is captured. The reason is that the effects of distortion of the positron wavefucntion are very important, and cannot be neglected. This distortion effect is most naturally taken into account in the rest frame of the nucleus.

The relationship between the Lorentz contraction factor associated with the relative velocity between the colliding nuclei, $\gamma$, and the collider energy per nucleon in $\mathrm{GeV}, E / A$, is given by $\gamma=2(1.0735 \times E / A)^{2}$, for $E / A \gg 1$. For example, at the RHIC, $E / A=100$, and $\gamma=2.3 \times 10^{4}$, while at the LHC, $E / A=3 \times 10^{3}$, and $\gamma \cong 2.07 \times 10^{7}$. According to refs. [2,17] the probability amplitude for pair production with capture for a collision with impact parameter $\mathbf{b}$ is given by

$$
a_{1 s t}=\frac{Z e}{i \pi} \int d^{2} q_{t} \frac{e^{i \mathbf{q}_{t} \cdot \mathbf{b}}}{q_{t}^{2}+(\omega / \mathrm{v} \gamma)^{2}} F(\mathbf{Q})
$$

where $\mathbf{q}_{t}$ is a transverse momentum vector with two components, $\mathbf{Q} \equiv\left(\mathbf{q}_{t}, \omega / \mathrm{v}\right)$ is a 3-component momentum vector with the third component equal to $\omega / \mathrm{v}$, and $\omega$ is the total energy used in the pair production. It is important to keep the relative velocity between the colliding nuclei, $\mathrm{v}$, in the right places for the moment (although $\mathrm{v} \cong c=1$ ) , since important combinations of 1 and $\mathrm{v}$ will lead to Lorentz factors $\gamma=\left(1-\mathrm{v}^{2}\right)^{-1 / 2}$ in subsequent steps of the calculation. In the case of K-shell capture $\omega=\varepsilon+m-I_{K}, I_{K}$ is the ionization energy of the K-shell electron, and $\varepsilon$ is the positron total energy $\varepsilon=\sqrt{p^{2}+1}$ (from now on we will use natural units, so that $\hbar=c=m=1$ ). The form factor $F(\mathbf{Q})$ is given in terms of the lepton transition current $j_{\mu}(r)=e \overline{\Psi^{(+)}} \gamma_{\mu} \Psi^{(-)}$as

$$
F(\mathbf{Q})=i e K_{\mu} \int d^{3} r \overline{\Psi^{(+)}}(\mathbf{r}) e^{i \mathbf{Q . r}} \gamma_{\mu} \Psi^{(-)}(\mathbf{r})
$$

where $K_{\mu} \equiv(0, \mathbf{K})$, with $\mathbf{K}=\left(\mathbf{q}_{t} / \omega, 1 / \mathrm{v} \gamma^{2}\right)$. For details of derivation of eqs. (112), see refs. [2,17]. The cross section is obtained by integrating the square of the expression (1) over all possible impact parameters:

$$
\sigma=\sum_{\text {spins }} \int\left|a_{1 s t}\right|^{2} d^{2} b=4 Z^{2} e^{2} \sum_{\text {spins }} \int d^{2} q_{t} \frac{|F(\mathbf{Q})|^{2}}{\left(\mathbf{Q}^{2}-\omega^{2}\right)^{2}} .
$$

For the positron wavefunction we use a plane wave and a correction term to account for the distortion due to the nucleus charge. The correction term is considered to be proportional to $Z e^{2}$. The wavefunction is then given by

$$
\Psi^{(+)}=N_{+}\left[\mathrm{v} e^{i \mathbf{p} \cdot \mathbf{r}}+\Psi^{\prime}\right],
$$

where $N_{+}$is a normalization constant. 
Only the Fourier transform of $\Psi^{\prime}$ will enter the calculation. This Fourier transform can be deduced directly from the Dirac equation for the positron in the presence of a Coulomb field of a nucleus:

$$
\left(\gamma^{0} \varepsilon+i \vec{\gamma} \cdot \vec{\nabla}+m\right) \Psi^{\prime}=-\frac{Z e^{2}}{r} \gamma^{0} \mathrm{v} e^{i \mathbf{p} \cdot \mathbf{r}}
$$

Applying on both sides of this equation the operator $\left(\gamma^{0} \varepsilon+i \vec{\gamma} \cdot \vec{\nabla}-m\right)$ we get

$$
\left(\Delta+\mathbf{p}^{2}\right) \Psi^{\prime}=-Z e^{2}\left(\gamma^{0} \varepsilon+i \vec{\gamma} \cdot \vec{\nabla}-m\right)\left(\gamma^{0} \mathrm{v}\right) \frac{e^{i \mathbf{p} \cdot \mathbf{r}}}{r}
$$

Multiplying by $e^{-i \mathbf{q} \cdot \mathbf{r}}$ and integrating over $d^{3} r$ we get

$$
\left(\mathbf{p}^{2}-\mathbf{q}^{2}\right) \Psi_{\mathbf{q}}^{\prime}=-Z e^{2}\left[2 \gamma^{0} \varepsilon-\vec{\gamma} \cdot(\mathbf{q}-\mathbf{p})\right]\left(\gamma^{0} \mathrm{v}\right) \frac{4 \pi}{(\mathbf{q}-\mathbf{p})^{2}}
$$

where we have used the identity $\gamma^{0}\left(\gamma^{0} \varepsilon-i \vec{\gamma} \cdot \vec{\nabla}+m\right)\left(\gamma^{0} \mathrm{v}\right)=0$. Thus,

$$
\overline{\Psi_{\mathbf{q}}^{\prime}} \equiv\left(\Psi_{\mathbf{q}}^{\prime}\right)^{\dagger} \gamma^{0}=4 \pi Z e^{2} \bar{v} \frac{2 \gamma^{0} \varepsilon+\vec{\gamma} \cdot(\mathbf{q}-\mathbf{p})}{(\mathbf{q}-\mathbf{p})^{2}\left(\mathbf{q}^{2}-\mathbf{p}^{2}\right)} \gamma^{0}
$$

We will need this result later to calculate $F(\mathbf{Q})$. The normalization constant $N_{+}$, which accounts for the distortion of the wavefunction to all orders is given by [2]

$$
N_{+}=\exp \left[\pi a_{+} / 2\right] \Gamma\left(1+i a_{+}\right), \quad a_{+}=Z e^{2} / \mathrm{v}_{+},
$$

where $v_{+}$is the positron velocity in the frame of reference of the nucleus where the electron is capture.

For the electron we use the distorted wavefunction described in ref. [19], i.e.,

$$
\Psi^{(-)}=\left[1-\frac{i}{2} \gamma^{0} \vec{\gamma} \cdot \vec{\nabla}\right] \mathrm{u} \Psi_{n o n-r}, \quad \text { where } \quad \Psi_{n o n-r}=\frac{1}{\sqrt{\pi}}\left(\frac{Z}{a_{H}}\right)^{3 / 2} \exp \left(-Z r / a_{H}\right)
$$

with $a_{H}=1 / e^{2}$ being the hydrogen Bohr radius.

Using the positron and the electron wavefunctions as given by eqs. (4) and (10), we get

$$
\begin{aligned}
F(\mathbf{Q}) & =i e N_{+} \int d^{3} r\left\{\overline{\mathrm{v}} e^{-i \mathbf{p} \cdot \mathbf{r}}+\overline{\Psi^{\prime}}\right\}\left(\gamma_{\mu} K^{\mu}\right) e^{i \mathbf{Q} \cdot \mathbf{r}} \\
\times & \left\{1-\frac{i}{2 m} \gamma^{0} \vec{\gamma} \cdot \vec{\nabla}\right\} \mathrm{u} \Psi_{n o n-r}(\mathbf{r}) \\
& \simeq i e N_{+} \int d^{3} r\left\{\overline{\mathrm{v}} e^{i(\mathbf{Q}-\mathbf{p}) \cdot \mathbf{r}}\left(\gamma_{\mu} K^{\mu}\right)\left[\left(1-\frac{i}{2 m} \gamma^{0} \vec{\gamma} \cdot \vec{\nabla}\right)\right] \mathrm{u} \Psi_{n o n-r}(\mathbf{r})\right. \\
+ & \left.\overline{\Psi^{\prime}} e^{i \mathbf{Q} \cdot \mathbf{r}}\left(\gamma_{\mu} K^{\mu}\right) \mathrm{u} \Psi_{n o n-r}(\mathbf{r})\right\}
\end{aligned}
$$

where in the last equation we neglected terms of highest orders in $Z \alpha$.

Integrating by parts yields

$$
F(\mathbf{Q}) \simeq i e N_{+}\left\{\overline{\mathrm{v}}\left(\gamma_{\mu} K^{\mu}\right)\left[\left(1+\frac{1}{2 m} \gamma^{0} \vec{\gamma} \cdot(\mathbf{Q}-\mathbf{p})\right) \mathrm{u}\left(\Psi_{n o n-r}\right)_{\mathbf{Q}-\mathbf{p}}\right]+\overline{\Psi_{\mathbf{Q}}^{\prime}}\left(\gamma_{\mu} K^{\mu}\right) \mathrm{u} \frac{1}{\sqrt{\pi}}\left(\frac{Z}{a_{H}}\right)^{3 / 2}\right\}
$$

Since the corrections are to first order in $Z \alpha$, we have replaced $\Psi_{n o n-r}(\mathbf{r})$ by its constant value $\left(Z / a_{H}\right)^{3 / 2}(1 / \sqrt{\pi})$ in the last term of eq. (12). If we would do the same in the first term it would be identically zero (except for $\mathbf{Q}=\mathbf{p}$ ). 
We need to keep the corrections in the wavefunctions at least to first order in $Z \alpha$; for $\varepsilon \gg 1$ these corrections yield a term of the same order as the plane-wave to the total cross section, as we shall see later.

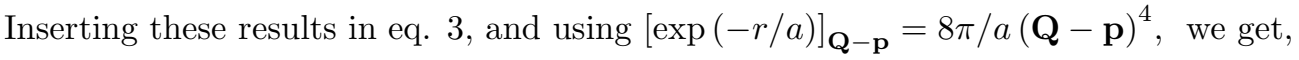

$$
F(\mathbf{Q})=i e N_{+} 8 \sqrt{\pi}\left(\frac{Z}{a_{H}}\right)^{5 / 2} \frac{\mathrm{v} A \mathrm{u}}{(\mathbf{Q}-\mathbf{p})^{2}},
$$

where

$$
A=a\left(\gamma_{\mu} K^{\mu}\right)+\left(\gamma_{\mu} K^{\mu}\right) \gamma^{0}(\vec{\gamma} \cdot \mathbf{b})+(\vec{\gamma} \cdot \mathbf{d}) \gamma^{0}\left(\gamma_{\mu} K^{\mu}\right)
$$

with

$$
\begin{aligned}
a & =\frac{1}{(\mathbf{Q}-\mathbf{p})^{2}}-\frac{\varepsilon}{\mathbf{Q}^{2}-\mathbf{p}^{2}}, \quad \mathbf{b}=\frac{\mathbf{Q}-\mathbf{p}}{2(\mathbf{Q}-\mathbf{p})^{2}}, \quad \text { and } \\
\mathbf{d} & =-\frac{\mathbf{Q}-\mathbf{p}}{2\left(\mathbf{Q}^{2}-\mathbf{p}^{2}\right)} .
\end{aligned}
$$

Inserting (13) into (3), with $\left|N_{+}\right|^{2}=2 \pi a_{+} /\left[\exp \left(2 \pi a_{+}\right)-1\right]$, performing the sum over spins with standard trace techniques, and using $p^{2} d p=\varepsilon p d \varepsilon(p \equiv|\mathbf{p}|)$, we get

$$
\begin{aligned}
\frac{d \sigma}{d \varepsilon d \Omega} & =\frac{64}{\pi a_{H}^{5} \mathrm{v}_{+}} \frac{Z^{8} e^{6} p}{\left[\exp \left(2 \pi Z e^{2} / \mathrm{v}_{+}\right)-1\right]} \int \frac{d^{2} q_{t}}{\left(\mathbf{Q}^{2}-\omega^{2}\right)^{2}} \frac{1}{(\mathbf{Q}-\mathbf{p})^{4}} \\
& \times\left\{(\varepsilon-1)\left[(\mathbf{b}-\mathbf{d})^{2} K^{2}+4(\mathbf{K} \cdot \mathbf{b})(\mathbf{K} \cdot \mathbf{d})\right]\right. \\
+ & \left.(\varepsilon+1) a^{2} K^{2}-2 a\left[2(\mathbf{p . K})(\mathbf{b} \cdot \mathbf{K})+\mathbf{p} \cdot(\mathbf{b}-\mathbf{d}) K^{2}\right]\right\} .
\end{aligned}
$$

In this relation we have used $\varepsilon_{-} \sim 1$, neglecting the electronic binding energy in the numerator of the expression inside the integral.

Using the definition of $K_{\mu}$, it is illustrative to separate the integrand of the above equation in terms of longitudinal and transverse components:

$$
\frac{d \sigma}{d \varepsilon d \Omega}=\frac{1}{\pi \omega} Z^{2} e^{2} \int_{0}^{\infty} d\left(q_{t}^{2}\right) \frac{q_{t}^{2}}{\left(\mathbf{Q}^{2}-\omega^{2}\right)^{2}}\left[\frac{d \sigma_{\gamma^{*} T}}{d \Omega}(\mathbf{Q}, \omega)+\frac{d \sigma_{\gamma^{*} L}}{d \Omega}(\mathbf{Q}, \omega)+\frac{d \sigma_{\gamma^{*} L T}}{d \Omega}(\mathbf{Q}, \omega)\right],
$$

where

$$
\begin{aligned}
& \frac{d \sigma_{\gamma^{*} T}}{d \Omega}(\mathbf{Q}, \omega)=\frac{64 \pi p}{a_{H}^{5} \omega \mathrm{v}_{+}} \frac{Z^{6} e^{4}}{\left[\exp \left(2 \pi Z e^{2} / \mathrm{v}_{+}\right)-1\right]} \frac{1}{(\mathbf{Q}-\mathbf{p})^{4}}\left\{(\varepsilon-1)\left[(\mathbf{b}-\mathbf{d})^{2}-\frac{\left(q_{t}-\mathbf{p} . \widehat{\mathbf{e}}_{t}\right)^{2}}{(\mathbf{Q}-\mathbf{p})^{2}\left(\mathbf{Q}^{2}-\mathbf{p}^{2}\right)}\right]\right. \\
& \left.+\quad(\varepsilon+1) a^{2}+2 a\left[\frac{\left(q_{t}-\mathbf{p} \cdot \widehat{\mathbf{e}}_{t}\right) \mathbf{p} \cdot \widehat{\mathbf{e}}_{t}}{(\mathbf{Q}-\mathbf{p})^{2}}-\mathbf{p} \cdot(\mathbf{b}-\mathbf{d})\right]\right\}, \\
& \frac{d \sigma_{\gamma^{*} L}}{d \Omega}(\mathbf{Q}, \omega)=\frac{64 \pi p \omega}{a_{H}^{5} q_{t}^{2} \gamma^{4} \mathrm{v}_{+}} \frac{Z^{6} e^{4}}{\left[\exp \left(2 \pi Z e^{2} / \mathrm{v}_{+}\right)-1\right]} \frac{1}{(\mathbf{Q}-\mathbf{p})^{4}}\left\{(\varepsilon-1)\left[(\mathbf{b}-\mathbf{d})^{2}-\frac{\left(\omega / \mathrm{v}-p_{z}\right)^{2}}{(\mathbf{Q}-\mathbf{p})^{2}\left(\mathbf{Q}^{2}-\mathbf{p}^{2}\right)}\right]\right. \\
& \left.+\quad(\varepsilon+1) a^{2}+2 a\left[\frac{p_{z}\left(\omega / \mathrm{v}-p_{z}\right)}{(\mathbf{Q}-\mathbf{p})^{2}}-\mathbf{p} \cdot(\mathbf{b}-\mathbf{d})\right]\right\}, \\
& \frac{d \sigma_{\gamma^{*} L T}}{d \Omega}(\mathbf{Q}, \omega)=\frac{128 \pi p}{a_{H}^{5} q_{t} \gamma^{2} \mathrm{v}_{+}} \frac{Z^{6} e^{4}}{\left[\exp \left(2 \pi Z e^{2} / \mathrm{v}_{+}\right)-1\right]} \frac{\left(\omega / \mathrm{v}-p_{z}\right)}{(\mathbf{Q}-\mathbf{p})^{6}} \\
& \times\left\{a\left[\left(\mathbf{p} . \widehat{\mathbf{e}}_{t}\right)+p_{z} \frac{\left(q_{t}-\mathbf{p} . \widehat{\mathbf{e}}_{t}\right)}{\left(\omega / \mathrm{v}-p_{z}\right)}\right]-(\varepsilon-1) \frac{\left(q_{t}-\mathbf{p} . \widehat{\mathbf{e}}_{t}\right)}{\left(\mathbf{Q}^{2}-\mathbf{p}^{2}\right)}\right\},
\end{aligned}
$$


In these equations $\widehat{\mathbf{e}}=\widehat{\mathbf{q}}_{t} / q_{t}$ is a unit vector in the transverse direction. The cross sections $\sigma_{\gamma^{*} T}, \sigma_{\gamma^{*} L}$, and $\sigma_{\gamma^{*} L T}$ are interpreted as the cross sections for pair production with capture by virtual transverse and longitudinal photons and a interference term, respectively. Note that the transverse and longitudinal directions are with respect to the beam axis, not with respect to the photon momentum, as is usually meant by this term. Only for $\gamma \gg 1$, this definition agrees with the definition of transverse and longitudinal virtual photons. However, this separation is very useful, as we will see next.

Now we turn to the relation to pair-production by real photons and the relation to the equivalent photon approximation.

\section{PAIR PRODUCTION WITH CAPTURE BY REAL PHOTONS: ROLE OF DISTORTION EFFECTS}

The cross section for pair production with capture real photons is given by [19]

$$
d \sigma_{\gamma}=2 \pi\left|V_{f i}\right|^{2} \delta(\omega-\varepsilon-1) \frac{d^{3} p}{(2 \pi)^{3}},
$$

where

$$
V_{f i}=-e \sqrt{\frac{4 \pi}{\omega}} \int d^{3} r \overline{\Psi^{(+)}}(\mathbf{r}) e^{i \vec{\kappa} \cdot \mathbf{r}}(\widehat{\mathbf{e}} \cdot \vec{\gamma}) \Psi^{(-)}(\mathbf{r})
$$

with $\vec{\kappa}=\widehat{\mathbf{z}} \omega$, where $\widehat{\mathbf{z}}$ is the unit vector along the photon incident direction, and $\widehat{\mathbf{e}}$ is photon polarization unit vector.

Using the positron and the electron wavefunctions from the previous section, and performing similar steps, we get

$$
V_{f i}=-e Z^{5 / 2} \frac{8 \pi \sqrt{2}}{a_{H}^{5 / 2} \omega^{1 / 2}} N_{+} \frac{\mathrm{v} B u}{(\vec{\kappa}-\mathbf{p})^{2}}
$$

where

$$
B=a_{\kappa}(\vec{\gamma} \cdot \vec{\kappa})+(\vec{\gamma} \cdot \vec{\kappa}) \gamma^{0}\left(\vec{\gamma} \cdot \mathbf{b}_{\kappa}\right)+\left(\vec{\gamma} \cdot \mathbf{d}_{\kappa}\right) \gamma^{0}(\vec{\gamma} \cdot \vec{\kappa})
$$

where $a_{\kappa}, \mathbf{b}_{\kappa}$, and $\mathbf{d}_{\kappa}$ are the quantities defined in eqs. 15 , but with $\mathbf{Q}$ replaced by $\vec{\kappa}=\widehat{\mathbf{z}} \omega$. Inserting these results in eq. 21 and summing over spins we get

$$
\begin{aligned}
\frac{d \sigma_{\gamma}}{d \Omega}(\omega) & =\frac{64 \pi e^{2} p}{a_{H}^{5} \omega \mathrm{v}_{+}} \frac{Z^{6} e^{2}}{\left[\exp \left(2 \pi Z e^{2} / \mathrm{v}_{+}\right)-1\right]} \frac{1}{(\vec{\kappa}-\mathbf{p})^{4}} \\
& \times\left\{(\varepsilon-1)\left[\left(\mathbf{b}_{\kappa}-\mathbf{d}_{\kappa}\right)^{2}-\frac{(\mathbf{p} . \widehat{\mathbf{e}})^{2}}{(\vec{\kappa}-\mathbf{p})^{2}\left(\kappa^{2}-p^{2}\right)}\right]\right. \\
+ & \left.(\varepsilon+1) a_{\kappa}^{2}+2 a_{\kappa}\left[\frac{(\mathbf{p} . \widehat{\mathbf{e}})^{2}}{(\vec{\kappa}-\mathbf{p})^{2}}-\mathbf{p} \cdot\left(\mathbf{b}_{\kappa}-\mathbf{d}_{\kappa}\right)\right]\right\} .
\end{aligned}
$$

We notice that the above equation can also be obtained from eq. 18 in the limit $q_{t} \rightarrow 0$ and $\mathrm{v} \longrightarrow c$. In this limit $\sigma_{\gamma^{*} L} \longrightarrow 0$, and $\sigma_{\gamma^{*} L T} \longrightarrow 0$, and $\sigma_{\gamma^{*} T}$ becomes the cross section for the production of pairs, with capture, by real photons.

Integrating eq. 25 over the azimuthal angle we get (without any further approximations!)

$$
\frac{d \sigma_{\gamma}}{d \theta}(\omega)=4 \pi^{2} \frac{Z^{6} e^{2}}{\left[\exp \left(2 \pi Z e^{2} / \mathrm{v}_{+}\right)-1\right]} \frac{e^{2} p^{3}(\varepsilon+2)}{a_{H}^{5} \omega^{4} \mathrm{v}_{+}} \frac{\sin ^{3} \theta}{(\varepsilon-p \cos \theta)^{4}}\left[\varepsilon-p \cos \theta-\frac{2}{\omega(\varepsilon+2)}\right]
$$

In the frame of reference of the nucleus, the angular distribution of the positrons is forward peaked, along the incidence of the photon. The higher the positron energy is, the more forward peaked the distribution becomes. In a 
collider, a Lorentz transformation of these results to the laboratory frame implies that all positrons are seen along the beam direction, within an opening angle of order of $1 / \gamma \ll 1$.

Integrating eq. 26 over $\theta$ we get

$$
\sigma_{\gamma}=\frac{8 \pi^{2} e^{2}}{a_{H}^{5} \mathrm{v}_{+}} \frac{Z^{6} e^{2}}{\left[\exp \left(2 \pi Z e^{2} / \mathrm{v}_{+}\right)-1\right]} \frac{p}{\omega^{4}}\left[\varepsilon^{2}+\frac{2}{3} \varepsilon+\frac{4}{3}-\frac{\varepsilon+2}{p} \ln (\varepsilon+p)\right]
$$

The relevance of the Coulomb distortion corrections can be better understood by using the high energy limit, $\varepsilon \gg 1$. From eq. (27) we get

$$
\sigma_{\gamma}=\frac{8 \pi^{2} e^{2}}{a_{H}^{5} \mathrm{v}_{+}} \frac{Z^{6} e^{2}}{\left.\exp \left(2 \pi Z e^{2} / \mathrm{v}_{+}\right)-1\right]} \frac{1}{\varepsilon}
$$

If we had used plane-waves for the electron and hydrogenic wave function for the positron in our calculation, without the corrections to order $Z \alpha$, the cross section for the pair production with capture by real photons would be given by

$$
\left[\frac{d \sigma_{\gamma}}{d \theta}(\omega)\right]_{P W A}=4 \pi \frac{Z^{5} e^{2} p}{a_{H}^{5} \omega^{4}} \frac{\sin \theta}{(\varepsilon-p \cos \theta)^{4}}
$$

the integral of which being

$$
\left[\sigma_{\gamma}\right]_{P W A}=\frac{8 \pi Z^{5} e^{2}}{a_{H}^{5}} \frac{p}{\omega^{4}}\left(3 \varepsilon^{2}+p^{2}\right)
$$

In the high energy limit:

$$
\left[\sigma_{\gamma}\right]_{P W A}=\frac{32 \pi Z^{5} e^{2}}{3 a_{H}^{5}} \frac{1}{\varepsilon}
$$

Using the approximation $Z \alpha \ll 1$ in eq. (28) we get

$$
\sigma_{\gamma}=\frac{4 \pi Z^{5} e^{2}}{a_{H}^{5}} \frac{1}{\varepsilon}
$$

which is different than eq. (31) by a factor $8 / 3$. This is important to show how the corrections of order $Z \alpha$ influence the result of the calculation. Not only they modify the results by inclusion of the correct normalization of the distorted positron wavefunction, $N_{+}$, but they also yield terms to the matrix elements for photo-production of the same magnitude as the terms of lowest order. The origin of the difference between the two results are the small distances which enter in the calculation of the matrix elements of eq. (22). These corrections are essential to account for their effects properly, and are enough to account for a good description of pair production with capture, as we will see in the next section. It is worhtwhile to mention that this equation confirms the findings of Sauter [20] for the annihilation of a positron with an electron in the K-shell of an atom. Using the detailed balance theorem that process can be related to the gamma production of electron-positron pairs with electron capture and the above equation is reproduced.

All positron variables in the above equations are expressed in the frame of reference of the ion where the electron is captured. Now the $\left(\mathbf{Q}^{2}-\omega^{2}\right)^{2}$ denominator of the first term inside the integral 17 shows that $q_{t} \cong \omega / \mathrm{v} \gamma$ is the relevant order of magnitude of $q_{t}$ in the integration. Thus, in all three virtual photon cross sections, 18, 19, and 20 we have $|\mathbf{Q}| \cong \omega / \mathrm{v} \cong \omega \gg \omega / \mathrm{v} \gamma$. The additional terms seem also to be independent from $\gamma$, what would imply that no extra $\gamma$ factor arise in these expressions. Thus, for large $\gamma, \sigma_{\gamma^{*} T} \cong \gamma^{2} \sigma_{\gamma^{*} L} \cong \gamma \sigma_{\gamma^{*} L T}$. This is confirmed by numerical calculations. Thus, for RHIC and LHC energies we can safely use

$$
\frac{d \sigma}{d \varepsilon d \Omega}=\frac{1}{\pi \omega} Z^{2} e^{2} \int_{0}^{\infty} d\left(q_{t}^{2}\right) \frac{q_{t}^{2}}{\left(\mathbf{Q}^{2}-\omega^{2}\right)^{2}}\left[\frac{d \sigma_{\gamma^{*} T}}{d \Omega}(\mathbf{Q}, \omega)\right] .
$$


The above discussion might seem convincing. But, it is still reasonable to check this result within a solvable model. This can be achieved by using plane-waves for the positron and hydrogenic waves for the electron, without the correction terms to order $Z \alpha$. In this case, we get

$$
F(\mathbf{Q})=4 i e \sqrt{\pi}\left(Z / a_{H}\right)^{5 / 2} \frac{\overline{\mathrm{v}}(\vec{\gamma} \cdot \mathbf{K}) \mathrm{u}}{(\mathbf{Q}-\mathbf{p})^{2}}
$$

which yields the cross section

$$
\frac{d \sigma}{d \varepsilon d \Omega}=\frac{1}{\pi \omega} Z^{2} e^{2} \int_{0}^{\infty} d\left(q_{t}^{2}\right) \frac{q_{t}^{2}}{\left(\mathbf{Q}^{2}-\omega^{2}\right)^{2}}\left[\frac{d \sigma_{\gamma^{*} T}}{d \Omega}(\mathbf{Q}, \omega)+\frac{d \sigma_{\gamma^{*} L}}{d \Omega}(\mathbf{Q}, \omega)\right]
$$

where the interference term is exactly zero, and

$$
\frac{d \sigma_{\gamma^{*} T}}{d \Omega}(\mathbf{Q}, \omega)=\frac{32 Z^{5} e^{2} p}{a_{H}^{5}} \frac{1}{(\mathbf{Q}-\mathbf{p})^{8}}, \text { and } \frac{d \sigma_{\gamma^{*} L}}{d \Omega}(\mathbf{Q}, \omega)=\frac{32 Z^{5} e^{2} p \omega^{2}}{a_{H}^{5} q_{t}^{2}}\left(\frac{1}{\gamma^{2} \mathrm{v}}\right)^{2} \frac{1}{(\mathbf{Q}-\mathbf{p})^{8}}
$$

The integral over $\Omega$ in the expressions above can be done analytically yielding

$$
\left\{\begin{array}{l}
\sigma_{\gamma^{*} T}(Q, \omega) \\
\sigma_{\gamma^{*} L}(Q, \omega)
\end{array}\right\}=\frac{128 \pi Z^{5} e^{2} p}{3 a_{H}^{5}} \frac{\left(\mathbf{p}^{2}+3 \mathbf{Q}^{2}\right)\left(3 \mathbf{p}^{2}+\mathbf{Q}^{2}\right)}{\left(\mathbf{Q}^{2}-\mathbf{p}^{2}\right)^{6}}\left\{\begin{array}{l}
1 \\
\omega^{2} /\left(q_{t} \gamma^{2} \mathrm{v}\right)^{2}
\end{array}\right\} .
$$

Using this result, the integral over $q_{t}^{2}$ in (35) can also be performed analytically but resulting in too long expressions to be transcribed here. We also note that the denominator $(\mathbf{Q}-\mathbf{p})^{2}$ in eq. (13) implies that $|\mathbf{p}| \cong|\mathbf{Q}| \cong \omega / \mathrm{v}$ and that the largest contribution to the integrals arise from the region with $(\mathbf{Q}-\mathbf{p})^{2} \cong \omega^{2} / \gamma^{2}$. Thus, in the plane wave approximation we really expect that $\sigma_{\gamma^{*} T}$ is by some power of $\gamma$ larger than the $\sigma_{\gamma^{*} L}$ cross section.

\section{TOTAL CROSS SECTIONS: COMPARISON WITH THE EQUIVALENT PHOTON APPROXIMATION}

The energy spectrum of the positrons can be obtained by integrating 33 over angles. The calculation can be done analytically if one neglects terms of order $\mathcal{O}(1 / \gamma)$. The expressions obtained were checked against the numerical results and we found that for RHIC energies the difference between the two results are only visible (of order of $1 \%$ ) for positron energies $\varepsilon \ll 1$. For positron energies $\varepsilon \gtrsim 1$ the differences are imperceptible. One gets

$$
\frac{d \sigma}{d \varepsilon}=\frac{32 \pi p}{a_{H}^{5} \omega \mathrm{v}_{+}} \frac{Z^{8} e^{6}}{\left[\exp \left(2 \pi Z e^{2} / \mathrm{v}_{+}\right)-1\right]}\left\{\frac{C D}{B^{6}}\left[\ln \left(\frac{B}{A}\right)-\frac{197}{60}\right]+\frac{3}{5} \frac{D}{B^{5}}+\frac{1}{5} \frac{C}{B^{5}}+\frac{3}{20} \frac{1}{B^{4}}\right\}
$$

where

$$
A=\frac{\omega^{2}}{\gamma^{2}}, \quad B=\omega^{2}-p^{2}, \quad C=3 \omega^{2}+p^{2}, \text { and } D=3 p^{2}+\omega^{2} .
$$

In ref. [2] analytical formulas were also derived for the differential and total cross section of pair production with K-shell electron capture. The cross sections were obtained by using Sommerfeld-Maue wave functions for the positron. Their result is

$$
\frac{d \sigma}{d \epsilon}=\frac{16 \pi p}{a_{H}^{5} \omega^{5} \mathrm{v}_{+}} \frac{Z^{8} e^{6}}{\left[\exp \left(2 \pi Z e^{2} / \mathrm{v}_{+}\right)-1\right]}\left[\frac{4}{3}+\frac{2 \varepsilon}{3}+\varepsilon^{2}-\frac{\varepsilon-2}{p} \ln (\varepsilon+p)\right] \ln \left(\frac{\gamma \delta}{\omega}\right),
$$

where $\delta=0.68108 \ldots$ is related to the Euler's number.

It is also worthwhile to compare the calculations with the equivalent photon approximation. The function 


$$
n(\gamma, Q)=\frac{1}{\pi} Z^{2} e^{2} \frac{q_{t}^{2}}{\left[q_{t}^{2}+(\omega / \gamma \mathrm{v})^{2}\right]^{2}}
$$

is called by "equivalent photon number", and is strongly peaked at $q_{t} \simeq \omega / \gamma \mathrm{v}$, which is very small for $\gamma \gg 1$. The equivalent photon approximation states that

$$
\frac{d \sigma}{d \varepsilon} \simeq \int_{0}^{q_{t}^{\max }} d\left(q_{t}^{2}\right) \frac{1}{\omega} n(\gamma, Q) \sigma_{\gamma^{*} T}\left(q_{t}=0, \omega\right)=\frac{1}{\omega} \sigma_{\gamma}(\omega) \int_{0}^{q_{t}^{\max }} d\left(q_{t}^{2}\right) n(\gamma, Q)
$$

where in the last equality one approximates $\sigma_{\gamma^{*} T}\left(q_{t}=0, \omega\right) \simeq \sigma_{\gamma}(\omega)$. The problem with the approximation is that the integral in eq. 42 diverges logarithmically. The approximation is only valid if we include a cutoff parameter $q_{t}^{\max }$, determined by the value of $q_{t}$ at which $\sigma_{\gamma^{*} T}(Q, \omega)$ drops to zero. For RHIC and LHC energies, we verified that the transverse virtual photon cross section drops to zero at $q_{t} \simeq \varepsilon$. Another hint for obtaining the appropriate cutoff value of $q_{t}$ is given in figure 2 where we plot the energy spectrum of the positron, $d \sigma / d \varepsilon$, for RHIC energies. We observe that the energy spectrum peaks at $\varepsilon \simeq 2-3$. We thus conclude that an appropriate value of the cutoff parameter is $q_{t}^{\max } \sim 1$. Inserting this value in eq. 42 we get the same result as 40 , except that in the last logarithm of that formula $\delta$ is replaced by $0.6065 \ldots$, an irrelevant change, in view of the large value of $\gamma$.

In figure 2 we compare our result, eq. 38 (solid line) with the approximation 40 (dashed line). We observe a good agreement, except at the low positron energies. This is an important result. The Sommerfeld-Maue wave functions (see [18] for a complete discussion about these wavefunctions) reproduce the exact Coulomb-Dirac wavefunction in the region relevant for the pair-production process. They account for the distortion effects in all orders. The result of figure 2 shows that, for pair production with K-shell capture, the approximation 1 , with \&, leads to very close agreement with that using Sommerfeld-Maue wavefunctions. That is, the most important part of the distortion is already taken into account by expanding the wavefunction to first-order in $Z \alpha$ and by using the correct normalization for the distorted wave 9 . We also note from figure 2 that the two results agree at large positron energies, $\varepsilon \gg 1$. That is expected, since distortion effects are not important at these energies and the outgoing positron is well described by plane-waves.

The total cross section can be obtained from eqs 38 and 40 by an integration over the positron energy. The integrations are performed numerically. In ref. [2] an approximate analytical result was obtained. Their result is

$$
\sigma=\frac{33 \pi}{10 a_{H}^{5}} Z^{8} e^{6} \frac{1}{\exp \left\{2 \pi Z e^{2}\right\}-1}[\ln (\gamma \delta / 2)-5 / 3] .
$$

The term [ $]^{-1}$ is the main efffect of the distortion of the positron wavefunction. It arises through the normalization of the continuum wavefunctions which accounts for the reduction of the magnitude of the positron wavefunction near the nucleus where the electron is localized (bound). Thus, the greater the $Z$, the less these wavefunctions overlap.

In figure 3 we compare the three results for RHIC energies, as a function of the atomic charge $Z$. The numerical integration of eqs. 38 and 40 yields essentially the same result, differing at most within $2 \%$ from each other. They are displayed by the solid curve. The dashed curve is the result of 43 . It agrees with the integrations of eqs. 38 and 40 for low $\mathrm{Z}(Z \lesssim 15)$ but disagrees by a factor 5 for large $\mathrm{Z}(Z \sim 80-90)$. The reason for this discrepancy is that eq. 43 was deduced in ref. [2] by probably neglecting the strong energy dependence (for large $Z$ ) of the exponential in the denominator of 38 which is a function of the positron velocity, $v_{+}$.

We note that the cross section 38 scales approximately with $Z^{7}$ for small values of $Z$, as also implied by eq. 43 . But, for large $Z$ 's there is not such a simple dependence. In our case, it is directly obtained from integration of eq. 38. The charge dependence of the capture cross sections have also been studied in refs. 12, 14 with slightly different results. For asymmetric systems, e.g., a projectile of charge $Z_{P}$ incident on a target of charge $Z_{T}$, the equation 38 should be changed by replacing $Z^{8}$ in the numerator by $Z_{P}^{2} Z_{T}^{6}$ and $Z$ in the exponential term of the denominator by $Z_{T}$. 
Equation 38 (and also 43) predicts a dependence of the cross section in the form $\sigma=A \ln \gamma_{c}+B$, where $A$ and $B$ are coefficients depending on the system and bombarding energy [2]. This dependence was used in the analysis of the experiment in ref. 16. In recent calculations, attention was given to the correct teatment of the distortion effects in the positron wavefunction [21]. In figure 4 we show the recent experimental data of ref. [16] compared to eq. 43 (dashed line) and to eq. 38. The data was obtained for $\mathrm{Pb}^{82+}$ at $33 \mathrm{TeV}$ impinging on several targets. The comparison with the experimental data is not fair since atomic screening was not taken into account here. When screening is present the cross sections will always be smaller at least by a factor 2-4 [2].

Using eq. 38 for $A u+A u$ beams at RHIC (100 GeV/nucleon) and $P b+P b$ beams at LHC (3 TeV/nucleon), we get $\sigma=229 \mathrm{~b}$ and $\sigma=562 \mathrm{~b}$, respectively. Other results can be easily obtained from a simple integration of eq. 38. In ref. [10] a numerical calculation was done making use of full Dirac wavefunctions, yielding $114 \mathrm{~b}$ for $A u+A u$ beams at RHIC. This is about 2 times larger than that obtaining using eq. 43 from ref. [2] and about 2 times smaller than our eq. 38. We assume that the reason for these diferences are that our wavefunctions are correct only to first order in $Z \alpha$. It might be that the inclusion of higher-order terms will change our results. However, the nice feature of our approach is that it provides an analytical description of the process and a direct connection with the photo-production cross sections.

\section{CONCLUSIONS}

We have calculated the cross sections for pair production with K-shell capture in peripheral collisions of relativistic heavy ions. The cross section can be separated into longitudinal and transverse components, with respect to the beam direction. For high energies, $\gamma \gg 1$, this corresponds to the usual meaning of longitudinal and transverse components of the photon. A very transparent and simple formulation is obtained using the lowest order corrections to the positron and electron wavefunctions. At ultra-relativistic energies, corresponding to the energies of relativistic heavy ion colliders (RHIC and LHC), the contribution of the longitudinal virtual photons to the total cross section vanishes. The remaining terms of the cross section can be factorized in terms of a virtual photon spectra and the cross section induced by real photons. However, the factorization depends on a cutoff parameter, which is not well defined. But, for $\gamma \gg 1$ a momentum cutoff parameter $q_{t} \sim 1$ reproduces extremely well the exact value of the cross section.

We note that the electron can be captured in any atomic orbit around one of the ions, the capture to the Kshell being the largest contribution. In ref. [2] it was shown that the contribution of capture to all other shells will contribute by an increase of $20 \%$ of the presently calculated cross section. Depending on the relevance of this process to peripheral collisions with relativistic heavy ions and on the accuracy attained by the experiments, more theoretical studies on the capture to higher orbits should be performed. At the present stage, it is important to notice that eq. 43, from ref. [2], is not a good approximation for large $\mathrm{Z}$ ions. The calculations developed in the present article were important to pinpoint the origin of this discrepancy and the validity of the equivalent photon approximation for $\gamma \gg 1$. It would be nice to show in a future study if the distortion corrections of higher order in $Z \alpha$ are indeed necessary to account for.

\section{Acknowledgments}

(*) John Simon Guggenheim Fellow.

This work was partially supported by the Brazilian funding agencies CNPq, CAPES, FUJB and MCT/FINEP/CNPQ(PRONEX) under contract No. 41.96.0886.00. E-mails: bertu@if.ufrj.br, dolci@if.ufrj.br. 
[1] C.A. Bertulani and G. Baur, Physics Today, March 1994, p. 22; and further references given there.

[2] C.A. Bertulani and G. Baur, Phys. Rep. 163 (1988) 299; Nucl. Phys. A505 (1989) 835

[3] G. Baur, K. Hencken and D. Trautman, J. Phys. G24 (1998) 1457

[4] S.R. Klein, LBL-PUB-45566, Los Alamos preprint server: \# physics/0005032

[5] G. Baur et al., Phys. Lett. B368 (1996) 351; G. Blanford et al., Phys. Rev. Lett. 80 (1998) 3037

[6] R. Anholt and U. Becker, Phys. Rev. A36 (1987) 4628

[7] U. Becker, J. Phys. B20 (1987) 6563.

[8] M.J. Rhoades-Brown, C. Bottcher and M.R. Strayer, Phys. Rev. A40 (1989) 2831

[9] K. Momberger, N. Grün and W. Scheid, Z. Phys. D18 1991) 133

[10] A.J. Baltz, M.J. Rhoades-Brown and J. Weneser, Phys. Rev. D18 (1991) 133; Phys. Rev. A47 (1993) $3444 ;$ Phys. Rev. A48 (1993) 2002; Phys. Rev. A50 (19 (1994) 4842

[11] K. Rumrich, G. Soff and W. Greiner, Phys. Rev. A47 1993) 215

[12] A. Aste, K. Hencken, D. Trautmann, and G. Baur, Phys. Rev. A50 (1994) 3930

[13] K. Momberger, A. Belkacem and A.H. Sorensen, Europhys. Lett. 32 (1995) 401

[14] C.K. Agger and A.H. Sorensen, Phys. Rev. A55 (1997) 402

[15] A.J. Baltz, Phys. Rev. Lett. 78 (1997) 1231

[16] H.F. Krause et al., Phys. Rev. Lett. 80 (1998) 1190

[17] C.A. Bertulani and G. Baur, Phys. Rev. D58 (1998) 034005

[18] H.A. Bethe and L.C. Maximon, Phys. Rev. 93 (1954) 768

[19] V.B. Berestetskii, E.M. Lifshitz, and L.P. Pitaevskii, Relativistic Quantum Field Theory, Part 1, 2nd ed. (Pergamon, New York, 1979)

[20] F. Sauter, Ann. Phys. (Leipzig) 9 (1931) 217; 11 (1931) 454

[21] H. Meier, et al., e-print nucl-th/0008020

\section{FIGURE CAPTIONS}

1. Diagram for pair production with capture in relativistic heavy ion colliders.

2. Energy spectrum of the emitted positron in pair production with capture for $100 \mathrm{GeV} /$ nucleon $P b+P b$ collisions at RHIC in the frame of reference of the nucleus where the electron is captured. The differential cross section is multiplied by the electron rest mass. The cross section is given in barns. The solid line is the exact calculation, while the dashed line is the analytical approximation, eq. 40 .

3. Total cross sections for pair production at RHIC as a function of the nuclear charge of the ions. The solid line is the exact calculation, and the dashed line is obtained from eq. 43 given in ref. [2]. 
4. Cross sections for pair production with electron capture for $\mathrm{Pb}^{82+}$ at $33 \mathrm{TeV}$ impinging on several targets with charge $Z$. The recent experimental data of ref. [16] is compared to eq. 43 (dashed line) and to eq. 38 (solid line). 


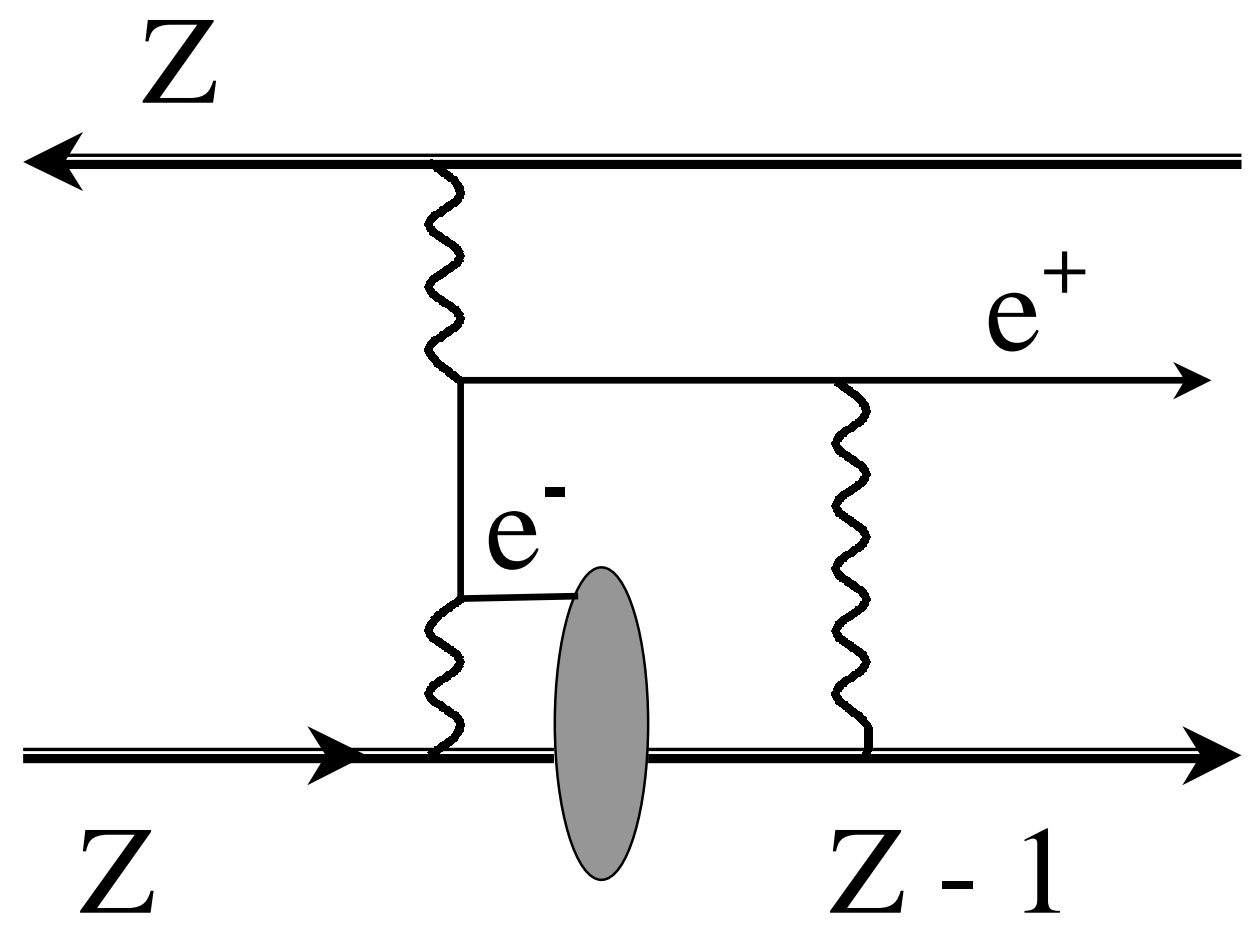




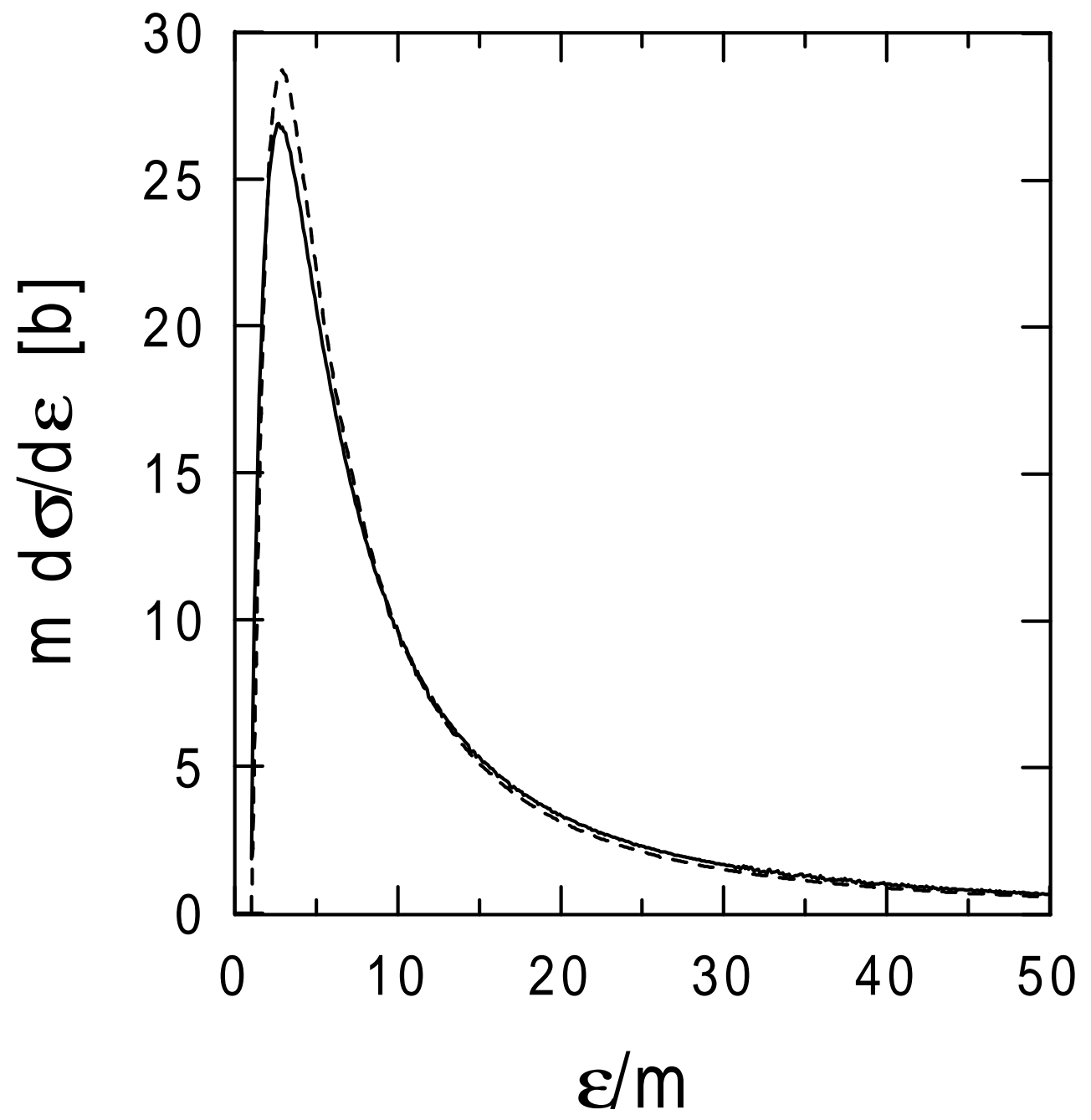




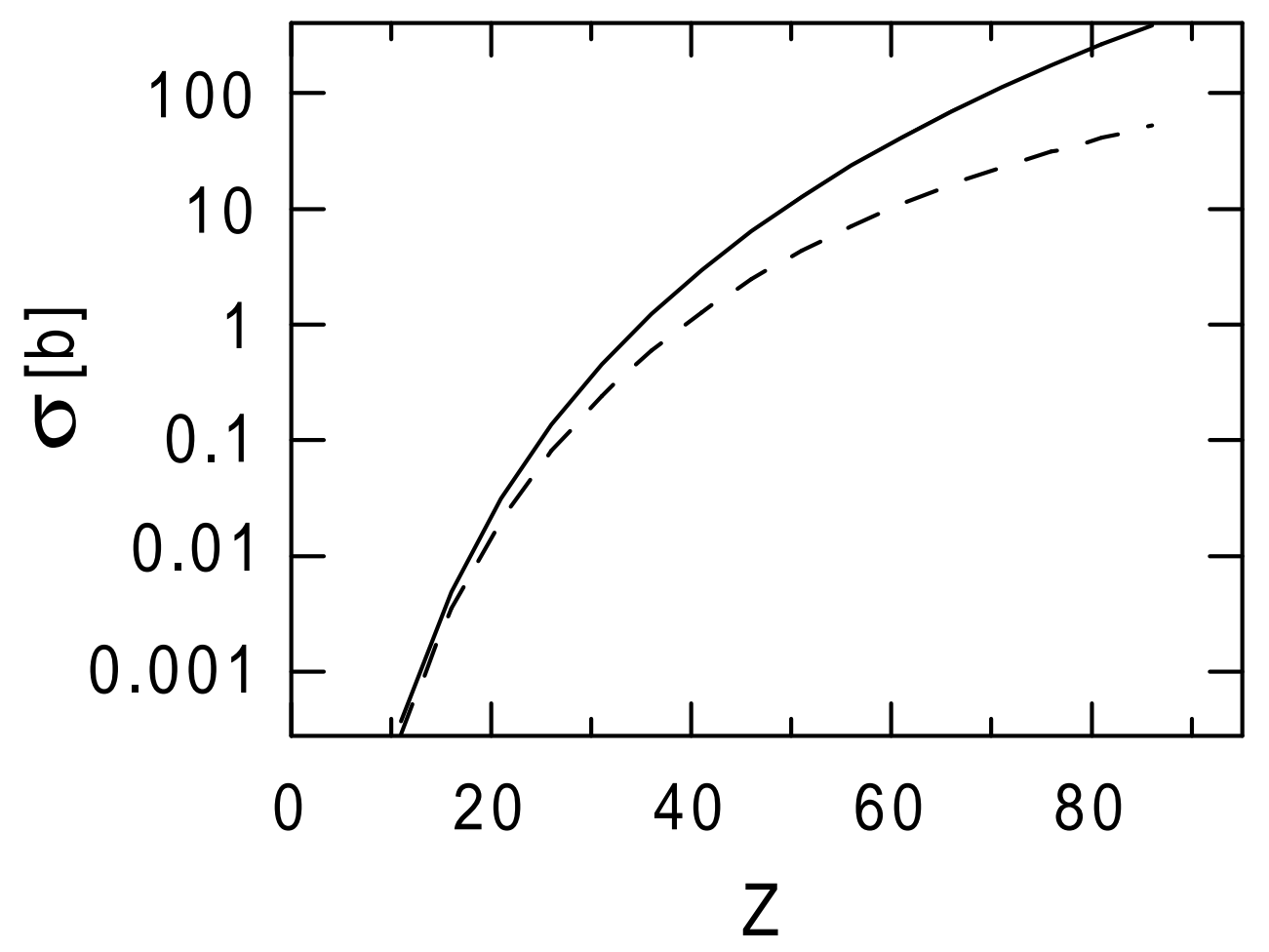




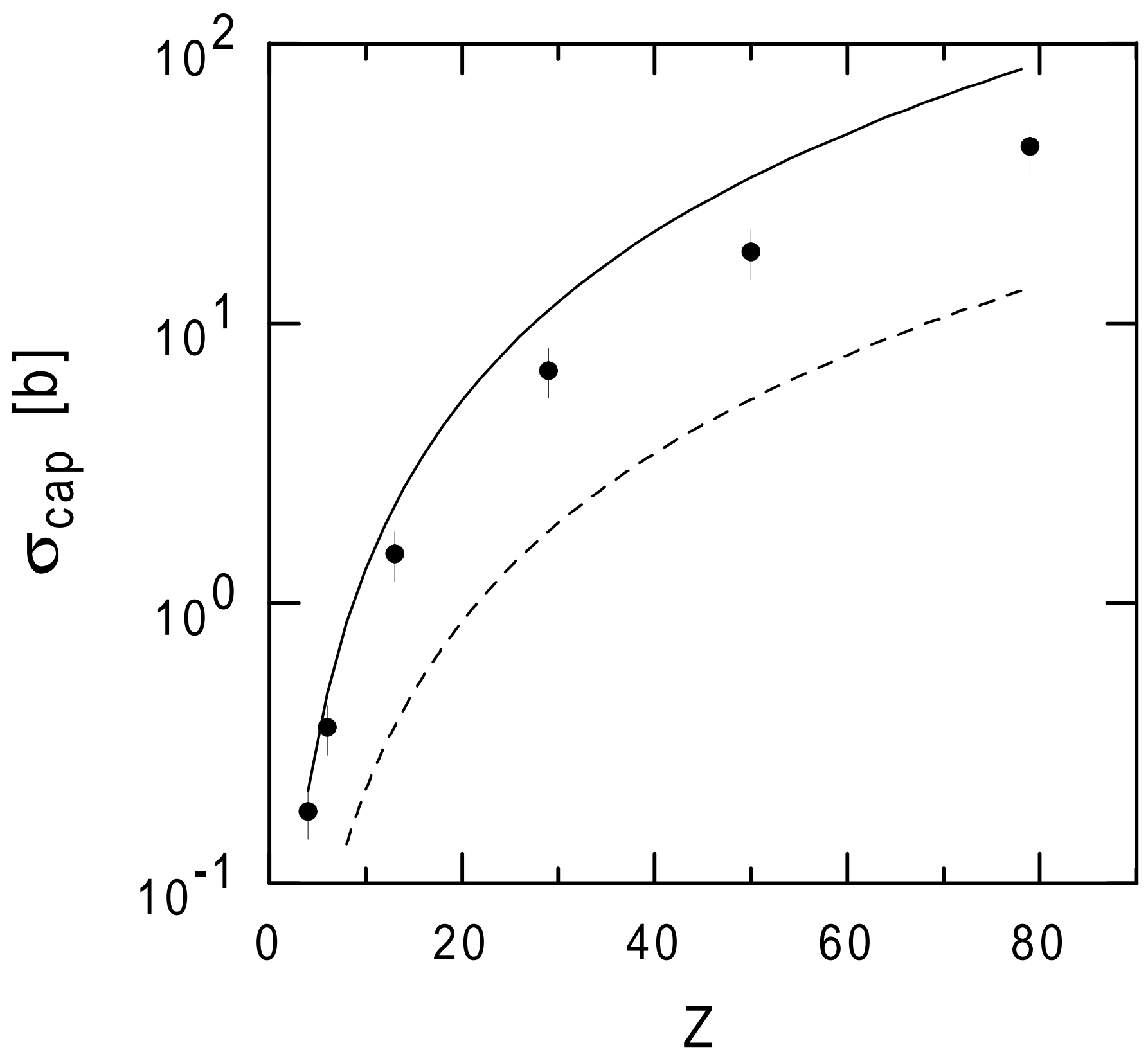

